\title{
Investigación de diseños de mezcla para producir Ultra High Performance Fiber Reinforced Concrete (UHPFRC) usando ANOVA
}

\section{Investigating mixing designs to produce Ultra High Performance Fiber Reinforced Concrete (UHPFRC) using ANOVA}

Rosangel Rojas Aguero ${ }^{1}$, Christa Korzenowski ${ }^{2}$, Jose Rafael Yepez Aguirre ${ }^{3}$, Ronaldo Beraldin ${ }^{2}$, Luiz Carlos Pinto da Silva ${ }^{2}$, Américo Campos Filho ${ }^{3}$

\footnotetext{
${ }^{1}$ Programa de Pós-graduação em Engenharia Civil - PPGEC/UFGRS, CEP: 90035 -190, Porto Alegre, Rio Grande do Sul, Brasil.

${ }^{2}$ Laboratório de Ensaios e Modelos Estruturais - LEME - PPGEC/UFGRS, CEP: 91501 -970, Porto Alegre, Rio Grande do Sul, Brasil.

${ }^{3}$ Programa de Pós-graduação em Engenharia Civil - PPGEC/UFGRS, CEP: 90035 -190, Porto Alegre, Rio Grande do Sul, Brasil.

e-mail: ckorzenowski@gmail.com, rosangel.rojas@ufrgs.br, jose.aguirre@ufrgs.br, ronaldoberaldin@ @otmail.com, lcarlos66@gmail.com, americo@ufrgs.br
}

\begin{abstract}
RESUMEN
Se presenta un estudio experimental para producir Ultra High Performance Fiber Reinforced Concrete (UHFPRC) incluyendo residuos industriales en la mezcla y determinar la resistencia a la compresión promedio. El estudio incluye arreglos factoriales para realizar análisis de varianza usando ANOVA. Los factores controlables fueron: (i) edad de la muestra; (ii) granulometría de la escoria; (iii) diseños de mezcla; (iv) porcentajes de fibras y (iv) tipo de curado. Los mayores valores de resistencia obtenidos fueron 138.12, 132.17, y $155.29 \mathrm{MPa}$, con sustituciones parciales de cemento por residuos industriales del 35,25 y $26 \%$ respectivamente. Los diseños de mezcla fueron fabricados aplicando un procedimiento simple, usando en la mezcla materiales como cemento, humo de sílice, escoria de alto horno, polvo de cuarzo y arena fina producidos en Brasil. También se adiciona el superplastificante diluido en agua y las fibras, las cuales se incorporan cuando la mezcla se convierte en una densa masa plástica. Los resultados demostraron que todos los factores controlables tienen influencia significativa en la variable de respuesta y que la probabilidad de que las variaciones en la resistencia sean atribuidas al error experimental es menor al $1 \%$.
\end{abstract}

Palabras-clave: UHPFRC, sustentable, resistencia a la compresión, ANOVA.

\section{ABSTRACT}

An experimental study is presented to produce Ultra High Performance Fiber Reinforced Concrete (UHPFRC) including industrial waste in the mix, and determine the average compressive strength. The study includes factor arrangements to perform analysis of variance using ANOVA. The controllable factors were: (i) age of the sample; (ii) granulometry of the slag; (iii) mix designs; (iv) percentages of fibers and (iv) thermal treatment in curing. The highest resistance values obtained were 138.12, 132.17 and $155.29 \mathrm{MPa}$, with partial substitutions of cement by industrial waste of 35,25 and $26 \%$ respectively. The mixing designs were manufactured using a simple procedure, using materials such as cement, silica fume, blast furnace slag, quartz powder and fine sand produced in Brazil. Also, the mixture includes the superplasticizer diluted in water and the fibers, which are incorporated when the mixture becomes a dense plastic mass. The results showed that all the controllable factors have significant influence on the response variable, and that the probability that the variations in resistance are attributed to the experimental error is less than $1 \%$.

Keywords: UHPFRC, sustainable, compressive strength, ANOVA. 


\section{INTRODUCCIÓN}

El concreto de ultra alto desempeño reforzado con fibras conocido por sus siglas en inglés como UHPFRC (Ultra High Performance Fiber Reinforced Concrete) es un material poco conocido en Latinoamérica, como ejemplos de la pesquisa que está siendo desarrollada tenemos a LOPEZ y OSORIO [1] en Colombia, NIETO [2] en Perú, FERNÁNDEZ [3] en Costa Rica, MAZIA [4] de la Universidade de São Paulo, TOLEDO et al. [5] de la Universidad Federal de Rio de Janeiro, MENDES [6] de la Universidad de Santa Catarina y el presente trabajo que está siendo desarrollado en la Universidad Federal de Rio Grande del Sur. En Norte América, Europa y Asia, códigos de diseños están establecidos y grandes obras de ingeniería han sido construidas, principalmente puentes y pasarelas, como puede ser detallado en RESPLENDINO y TOULEMONDE [7] o en LOPEZ et al. [8].

Uno de los más notables aspectos que caracteriza a este tipo de material es que desarrolla una resistencia a la compresión que supera los $150 \mathrm{MPa}$, producto de la elaboración de una densa matriz con mínima porosidad, sin agregado grueso y con la presencia de fibras. Alcanzar esa alta resistencia sin la utilización de un minucioso programa de empaquetamiento de partículas es un verdadero desafío, que bien vale la pena asumir si el objetivo es producir una mezcla de más fácil elaboración y al alcance de cualquiera que disponga de equipos simples de construcción.

El UHPFRC ha permitido construir vigas con una sección transversal más esbelta comparada al concreto tradicional, bajo las mismas cargas de servicio y con la misma distancia entre apoyos. Esto permite disminuir la carga de la estructura, construir fundaciones con menor geometría y por tanto producir ahorro en la construcción de la obra. Si bien el costo inicial puede parecer mayor, se ha demostrado que el costo total de la obra puede ser disminuido entre un 10 a un 15\%, FEHLING, et al. [9]. Otro aspecto que es importante señalar y que a primera vista parece obvio es pensar que mayores cantidades de cemento producirán proporcionalmente mayores cantidades de contaminantes al ambiente, sin embargo, substituciones parciales de cemento por residuos industriales, como escoria de alto horno o GGBS (Ground Granulated Blast-furnace Slag) y humo de sílice (Silica Fume), permiten fabricar mezclas UHPFRC menos invasivas al ambiente, incluyendo de este modo a la sustentabilidad en el diseño estructural para acompañar a los tradicionales criterios de resistencia, durabilidad y seguridad.

El objetivo de éste estudio es producir y analizar varios diseños de mezclas hasta alcanzar el ultra alto desempeño del concreto reforzado con fibras, incluyendo residuos industriales en su composición como sustituyentes parciales del cemento. La resistencia a la compresión será determinada atendiendo a las recomendaciones de la Associação Brasileira de Normas Técnicas (ABNT), ABNT NBR-5739:2008 [10] y ABNT NBR-7215:1996 [11]. Será aplicado un análisis de varianza usando ANOVA como una herramienta estadística para estudiar los resultados y determinar la significancia de cada factor controlable sobre la variable de respuesta, que en nuestro caso es la resistencia a la compresión.

\section{REVISIÓN BIBLIOGRÁFICA}

LARRARD y SEDRAN [12] fueron unos de los primeros en usar el término Ultra-High Performance Concrete (UHPC) para referirse a un mortero fluido con una óptima densidad. Utilizaron el Modelo de Sólidos en Suspensión o SSM (Solid Suspension Model), como modelo de empaquetamiento de partículas. Trabajaron con una relación agua/aglomerantes (a/a) que varía entre de 0,106 hasta 0,178. Alcanzaron una resistencia de $237,90 \mathrm{MPa}$ al aplicar sistema de curado que incluye tratamiento térmico a $90^{\circ} \mathrm{C}$ por 4 días y posteriormente a temperatura ambiente.

En la década del 90, Richard y Cheyrezy [13] aplicaron un cierto número de principios básicos de diseño referentes a la composición, mezcla y posterior curado térmico del concreto, para obtener un material dúctil con ultra alto desempeño denominado Reactive Powder Concrete (RPC). Emprendieron un programa de pesquisa para desarrollar concretos con resistencias a la compresión entre 200 y $800 \mathrm{MPa}$, a través de mejoras en la homogeneidad de la mezcla mediante la eliminación del agregados grueso, mejoras de la densidad compactada por optimización de la mezcla granular y mejoraron la microestructura mediante tratamiento térmico en el curado, la ductilidad fue obtenida por la adición de fibras cortas de acero a la mezcla y mantuvieron los procedimientos lo más cerca posible de la práctica existente.

Rossi et al. [14] obtuvieron $205 \mathrm{MPa}$ de resistencia a la compresión usando mezclas de concreto con diferentes porcentajes y tipos de fibras de acero, incluyeron tratamiento térmico en el curado de los cuerpos de prueba. LIM y NAWY [15] alcanzaron $121 \mathrm{MPa}$ usando probetas de concreto cúbicas de $100 \mathrm{~mm}$ de lado, conteniendo $1,5 \%$ de fibras, fueron almacenados en cámara húmeda para el curado sin aplicar tratamiento térmico. HABEL et al. [16] obtuvieron $168 \mathrm{MPa}$ utilizando cemento con bajo contenido de C3A (Aluminato tricálcico) y fibras cortas de acero en la mezcla, con una relación a/a de 0,14 y con una relación 
agua/cemento de 0,18. Graybeal [17] alcanzó $193 \mathrm{MPa}$ de resistencia a la compresión a los 28 días usando especímenes con tratamiento térmico en el curado, en esos casos se observó una estabilización del valor de la resistencia a las 48 horas de curado, también ensayó especímenes sin tratamiento térmico alcanzando los 126 $\mathrm{MPa}$, en esos casos se observó que ganancias importantes en la resistencia continuaban después de ocho semanas de vaciado. Thanh [18] desarrolló un extenso trabajo experimental estudiando la influencia de la relación a/c, tratamiento térmico en el curado, influencia de la escoria, humo de sílice, cenizas pulverizadas, contenido de fibras, efecto del tamaño y tipo de agregado en el diseño de mezcla. Alcanzó resistencia a la compresión en rangos que varían entre 185,90 y 182,20 MPa.

YANG et al. [19] enfocaron su pesquisa en la disminución de costos de producción y en la utilización en obra (in situ) de la mezcla. Usaron dos diferentes tipos de agregados para sustituir la arena de sílice, primero incorporaron vidrio roto reciclado de la producción de vidrio o Recycled Glass Cullet (RGC) y segundo lugar usaron dos tipos de arenas locales, aplicaron tratamiento térmico en el proceso de curado, ensayaron a la compresión cubos de concreto de $50 \mathrm{~mm}$. Al final demostraron que usando el vidrio la resistencia disminuyó un $20 \%$ y usando las arenas locales no se obtuvo diferencia significativa en la resistencia comparada al uso de arena de sílice. Alcanzaron entre 160 y180 MPa usando arenas locales curadas a $90^{\circ} \mathrm{C}$ y 100 y 140 $\mathrm{MPa}$ a $20^{\circ} \mathrm{C}$ y usando RGC alcanzaron entre 150 y160 $\mathrm{MPa}$ a $90^{\circ} \mathrm{C}$ y entre 80 y $120 \mathrm{MPa}$ sin tratamiento térmico en el curado, en todos los casos éste autor usó una relación a/a de 0,15 .

TUAN et al. [20] incorporaron a la mistura de concreto ceniza molidas de cáscara de arroz o Rice Husk Ash (RHA) como sustituyente del humo de sílice. Utilizaron el modelo de empaquetamiento de partículas desarrollado por Larrard y SEDRAN [12] para optimizar la granulometría de la mistura. Demostraron que a medida que aumenta el tamaño promedio de la RHA la resistencia a la compresión disminuye. Alcanzaron $150 \mathrm{MPa}$ con un tamaño máximo del grano de $8 \mu \mathrm{m}$ y $20 \%$ de ese material respecto al peso del cemento. Hassan et al. [21] desarrollaron una metodología simple para la determinación de la resistencia a la compresión en probetas de concreto con fibras, pudiendo medir las deformaciones tanto en la fase elástica como en la inelástica del ensayo. Alcanzaron 121,32 y 150,56 MPa a los 28 días con y sin fibras respectivamente, emplearon el diseño de mezclas propuesto por Thanh [18] que incluye además del cemento, el humo de sílice y la GGBS como materiales aglomerantes. La relación a/a usada fue de 0,17 y la de a/c de 0,24. Usaron tratamiento térmico en el agua de curado a $90^{\circ} \mathrm{C}$ por 48 horas y posteriormente a temperatura ambiente hasta el día de la prueba.

Toledo et al. [5] alcanzaron $160 \mathrm{MPa}$ de resistencia a la compresión, maximizando la compactación de la mezcla granular basados en criterios de optimización en la distribución del tamaño de los granos y en el empaquetamiento de partículas. Usaron cemento (Bast furnace cement type III/40) y como aditivos minerales la Wollastonita y el humo de sílice. YU et al. [22] emplearon el modelo modificado de empaquetamiento de partículas de Andreasen \& Andersen en la mezcla, para alcanzar 94,2 y 148,6 MPa a los 28 días, sin y con fibras en la matriz de concreto respectivamente. WU et al. [23] consiguieron $118 \mathrm{MPa}$ a los 28 días usando $25 \%$ de humo de sílice en la mezcla de concreto, usaron una relación a/a de 0,18 y una relación a/c de 0,21. Lampropoulos et al. [24] ensayaron probetas cúbicas de $100 \mathrm{~mm}$ de lado a la compresión, con una velocidad de carga de $0,0007 \mathrm{~mm} / \mathrm{seg}$, alcanzando $164 \mathrm{MPa}$ a los 28 días en los especímenes que incluían 3\% de fibras cortas de acero en la mistura y aplicando tratamiento térmico de curado, los especímenes fueron sumergidos en agua cn una temperatura inicial de $90{ }^{\circ} \mathrm{C}$ por tres días y posteriormente a temperatura ambiente.

LIU et al. [25] agregaron a la mistura de concreto basalto triturado como agregado grueso con diámetros entre 5 a $20 \mathrm{~mm}$ y arena de rio con tamaño máximo de $5 \mathrm{~mm}$, alcanzaron una resistencia a la compresión de 99,70 MPa sin fibras y 143,6 MPa usando 2,5\% de fibras cortas de acero en probetas cúbicas de $100 \mathrm{~mm}$ de lado; trabajaron con una relación a/a de 0,18 y variaron las cantidades de agregado grueso, superplastificante y fibras de acero. KANG et al. [26] investigaron la resistencia a la compresión en probetas cúbicas de $50 \mathrm{~mm}$ de lado usando como materiales aglomerantes el cemento y el humo de sílice de zirconio, usaron también aditivos para reducir la retracción autógena, fibras de acero y fibras híbridas; la mayor resistencia en los especímenes híbridos fue obtenida combinando $1,5 \%$ de fibras de acero con $0,5 \%$ de fibras de polivinilo, obteniendo en ese caso 147,49 MPa.

LEE et al. [27] desarrollaron estudios de resistencia a la compresión usando probetas cilíndricas y también estudiaron la resistencia a la tracción usando elementos planos. Trabajaron con dos tipos de mezcla con y sin fibras, alcanzando valores de 127,38 MPa sin fibras y 157,46 MPa con fibras. Hoang y Fehling [28] concluyeron en su pesquisa que no existen cambios importantes en la resistencia a la compresión ni en el módulo de elasticidad por la inclusión de fibras de acero en pruebas de resistencia a la compresión usando cilindros de $15 \mathrm{~cm}$ de diámetro y $30 \mathrm{~cm}$ de altura, lo cual contradice a la mayoría de los estudios consultados. Los autores alcanzaron 216,66 y 211,69 MPa sin y con 1,5\% de fibras respectivamente. Kahanji et al. [29] alcanzaron 97,20 MPa usando $1 \%$ de fibra con curado de los especímenes en agua a $20^{\circ} \mathrm{C}$ y $154,60 \mathrm{MPa}$ con 
curado en agua a $90^{\circ} \mathrm{C}$, ambas por 7 días y posteriormente todos los especímenes fueron almacenados en sala húmeda hasta el día de la prueba. WU et al. [30] fabricaron una mezcla de concreto sin fibras y cinco mezclas con fibras para evaluar las propiedades estáticas y dinámicas del concreto sometido a compresión, alcanzando 98,30 y 143,6 MPa respectivamente. Alsalman et al. [31] alcanzaron una resistencia a la compresión máxima de 118,6 MPa en especímenes sin fibras y sin tratamiento térmico en el curado, usando materiales comerciales locales, también trabajaron con especímenes incluyendo tratamiento térmico en el curado, alcanzando $127,38 \mathrm{MPa}$ sin fibras y $157,46 \mathrm{MPa}$ con fibras.

\section{MATERIAIS E MÉTODOS}

El trabajo experimental mostrado en este estudio fue desarrollado en el Laboratorio de Ensayos y Modelos Estructurales (LEME) de la Universidad Federal de Rio Grande del Sur (UFRGS). Es una pesquisa por etapas, donde los resultados que van siendo obtenidos sirven de base para la toma de decisiones en las etapas subsiguientes. La Figura 1 muestra las tres etapas experimentales del trabajo, en cada fase fueron desarrollados diseños de mezclas para generar concretos con ultra alta resistencia. Factores controlables, sus niveles y las combinaciones entre ellos fueron definidos. Se fabricaron en total 342 cuerpos de prueba cilíndricos para los cuales se determinó la resistencia a la compresión uniaxial. Posteriormente un análisis de variancia usando ANOVA se llevó acabo, para determinar si algún factor controlable o alguna interacción entre factores tienen influencia significativa en la variable de respuesta.

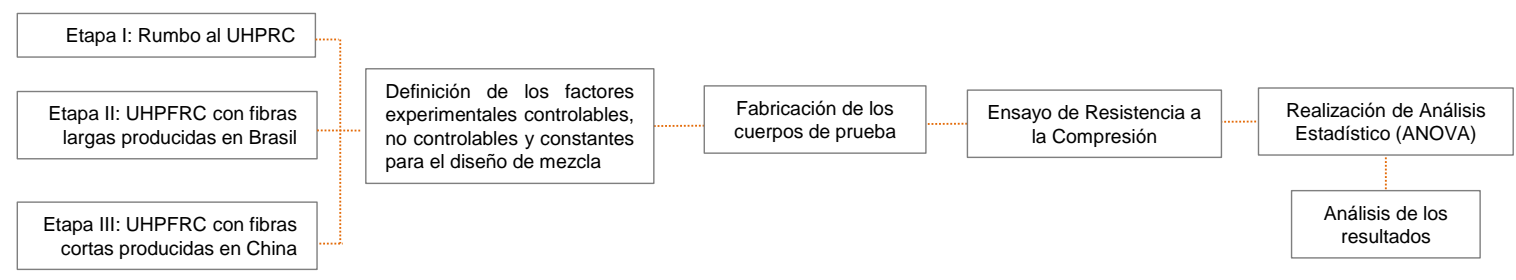

Figura 1: Esquema del trabajo. Fuente propia.

Los materiales a usar en los diseños de mezcla son: (i) cemento, de tipo CP-V con alta resistencia inicial; (ii) humo de sílice del tipo ND SILMIX fabricado por Dow Corning Metais do Pará Indústria e Comércio Ltda; (iii) escoria de alto horno o GGBS, que es un residuo de la producción del hierro tipo Gusa y fue donado por la Siderúrgica ArcelorMittal Tubarão ubicada en Espírito Santo, se trabajó con seis granulometrías diferentes; (iv) polvo de cuarzo, de uso comercial; (v) arena fina, de uso comercial; (vi) superplastificante, solución a base de policarboxilatos en medio acuoso (ViscoCrete 3535) donado por la empresa SIKA, para ajustar la trabajabilidad del concreto y es mezclado con agua para ser colocado en la mezcla; (vi) fibras largas de acero, donadas por la empresa Belgo, las cuales poseen una longitud de 3,5 cm y un factor de forma de 65; (vii) fibras de polipropileno donadas por la empresa Sika, con una longitud de 1,5 cm y sección plana texturizada de $2 \mathrm{~mm}$ de ancho. La Tabla 1 presenta los diseños de mezcla usados en este estudio.

Tabla 1: Diseños de mezclas Etapas I, II y III $\left(\mathrm{kg} / \mathrm{m}^{3}\right)$. Fuente propia.

\begin{tabular}{|c|c|c|c|c|c|c|c|c|}
\hline & \multicolumn{3}{|l|}{ ETAPA - I } & \multicolumn{4}{|l|}{ ETAPA - II } & \multirow{2}{*}{\begin{tabular}{|l} 
ETAPA - III \\
UHPFRC-1
\end{tabular}} \\
\hline Material $\left(\mathrm{kg} / \mathrm{m}^{3}\right)$ & UHPFRC & 1 UHPFR & UHPFRC-3 & UHPFRC & UHPFRC & UHPFRC & UHPFRC-4 & \\
\hline Cimento & \begin{tabular}{|l|}
597,00 \\
\end{tabular} & 657,00 & 776,10 & 776,00 & 854,00 & 895,00 & 895,00 & 955,00 \\
\hline \multirow{2}{*}{ GGBS } & 537,30 & 418,00 & 179,10 & 179,00 & 119,00 & 60,00 & 60,00 & 263,00 \\
\hline & GGBS-1 & GGBS-2 & GGBS-3 & GGBS-4 & GGBS-4 & GGBS-4 & GGBS-5 & GGBS-6 \\
\hline Silica Fume & \begin{tabular}{|l|}
59,70 \\
\end{tabular} & 119,00 & 238,80 & 239,00 & 239,00 & 238,80 & 238,80 & 119,00 \\
\hline Pó de Quartzo & 0,00 & 0,00 & 0,00 & 0,00 & 0,00 & 0,00 & 0,00 & 119,00 \\
\hline SS & $1.051,00$ & $1.051,00$ & $1.051,00$ & $1.051,00$ & $1.051,00$ & $1.051,00$ & $1.051,00$ & 788,00 \\
\hline Superplastificante & 40,00 & 40,00 & 40,00 & 40,00 & 40,00 & 40,00 & 40,00 & 40,00 \\
\hline Àgua & 185,00 & 185,00 & 185,00 & 185,00 & 185,00 & 185,00 & 185,00 & 185,00 \\
\hline Fibra Belgo 1\% & 0,00 & 0,00 & 0,00 & \begin{tabular}{|l|}
78,50 \\
\end{tabular} & 78,50 & 78,50 & 78,50 & 0,00 \\
\hline Fibra Sika 1,5\% & 0,00 & 0,00 & 0,00 & 9,00 & 9,00 & 9,00 & 9,00 & 13,50 \\
\hline Fibras Chinas 1\% & 0,00 & 0,00 & 0,00 & 0,00 & 0,00 & 0,00 & 0,00 & 78,50 \\
\hline Fibras Chinas 1,5\% & 0,00 & 0,00 & 0,00 & 0,00 & 0,00 & 0,00 & 0,00 & 117,75 \\
\hline
\end{tabular}

La fabricación de la mezcla y de los cuerpos de prueba fue realizada según las estipulaciones de las 
normas ABNT NBR 7215:1996 [11] ABNT NBR 5738:2003 [32] y también siguiendo el procedimiento propuesto por THANH [18] en la Liverpool University para concretos con ultra alta resistencia. Primeramente los materiales son pesados y colocados en una mezcladora para argamasa siguiendo el siguiente orden: humo de sílice, cemento, escoria de alto horno, polvo de cuarzo (opcional) y arena de sílice (Figura 2-a). Los materiales secos se mezclan por aproximadamente 5 minutos antes de añadir el superplastificante previamente mezclado con agua, (Figura 2-b). Todos los materiales son mezclados entre 10 a 15 minutos más (Figura 2-c), hasta que la mezcla se convierta en una húmeda pasta de concreto, (Figura 2-d). En ese estado la mezcla está lista y es entonces cuando las fibras son adicionadas (opcional) y se mezcla todo por 2 minutos más (Figura 2-e). Se procede entonces a vaciar la masa de concreto en los moldes cilíndricos (Figura 2-f), los cuales deben ser compactados sobre una mesa vibratoria por 1 minuto.

Los cuerpos de prueba deben ser almacenados y cubiertos con una manta plástica por 24 horas, luego de ese tiempo los especímenes son divididos para aplicar o no el tratamiento térmico en el curado, el cual consistió en sumergir los cuerpos de prueba en baño térmico con temperatura de $60^{\circ} \mathrm{C}$ por 24 horas y luego a $90{ }^{\circ} \mathrm{C}$ por 24 horas adicionales, es decir, cuando los cuerpos de prueba son almacenados en la cámara húmeda a temperatura de $20 \pm 3{ }^{\circ} \mathrm{C}$ tienen una edad de cinco días, y permanecen allí hasta el día del ensayo. El procedimiento seleccionado para aplicar o tratamiento térmico fue basado en el estudio de GRAYBEAL [17] y THANH [18], adaptándolo a las limitaciones propias del laboratorio LEME. En [33] se indica el link a seguir para visualizar un video que muestra el proceso de fabricación del UHPFRC.

(a)

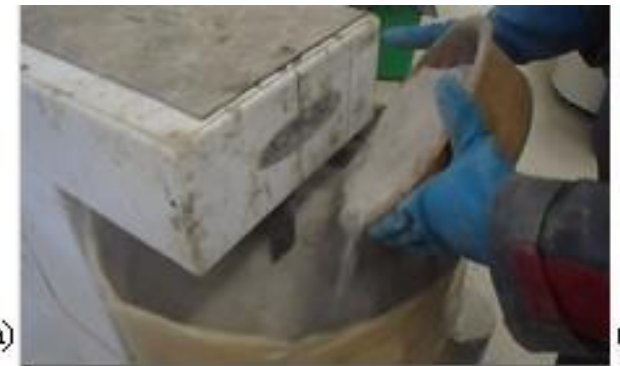

(c)

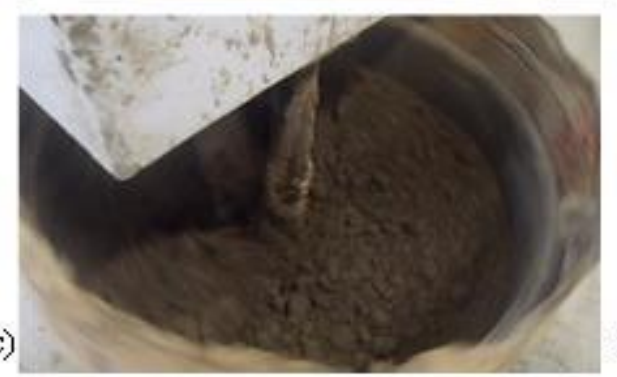

(e)

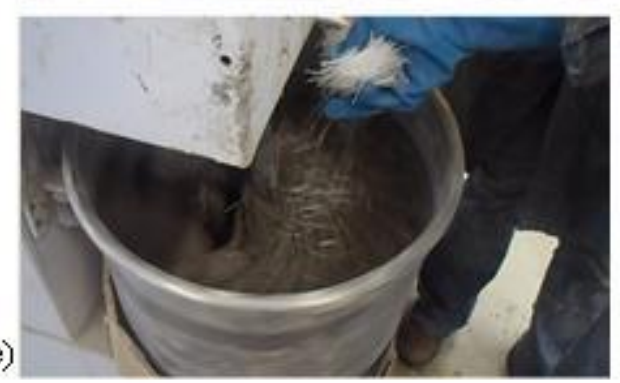

(b)

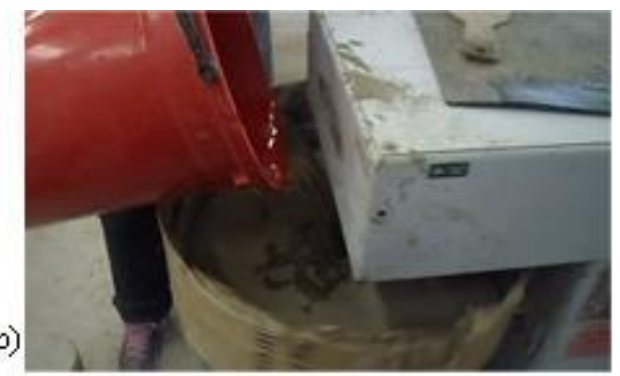

(d)
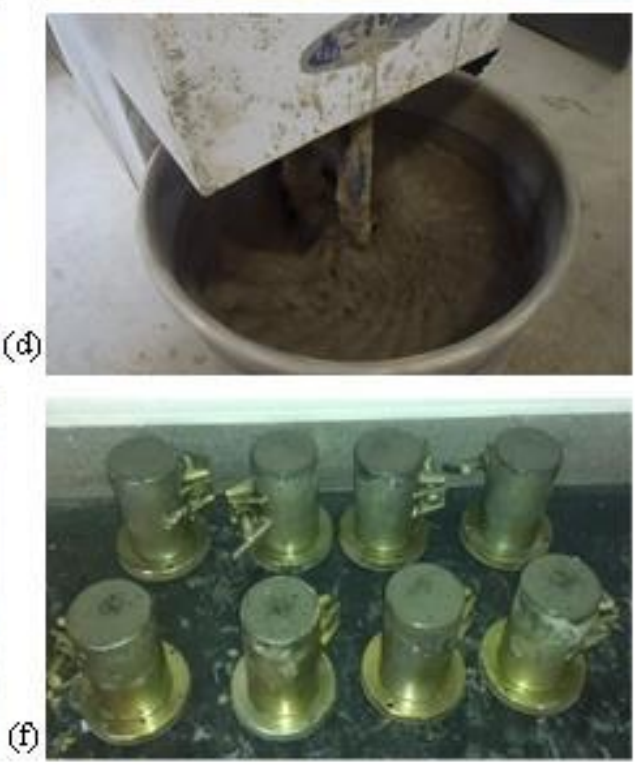

Figura 2: Proceso de fabricación del UHPFRC. Fuente propia.

En la II etapa experimental fue usado el programa EMMA [34], el cual es utilizado para densificar la matriz de concreto. Este programa está disponible online para uso gratuito, con el cual es posible obtener una curva granulométrica experimental del diseño de mezcla por la introducción de la granulometría de cada material separadamente. Al final esa curva es comparada con la curva teórica basada en el modelo de empaquetamiento teórico de Andreassen y Andersen modificado, y a partir de esa comparación es posible determinar el exceso o la falta de ciertas granulometrías para alcanzar la máxima densificación de la matriz y por ende el incremento de la resistencia a la compresión.

El ensayo de resistencia a la compresión uniaxial se realiza usando una prensa hidráulica (Universal Testing Machine) marca Shimadzu, modelo UH-F2000kNA con una capacidad de 2000 kN. Previo al ensayo 
las caras planas de los cilindros son niveladas usando una rectificadora. Luego se mide la altura y el peso de cada cuerpo de prueba para verificar la necesidad de aplicar algún factor de corrección en la resistencia, todo el procedimiento sigue las estipulaciones de la norma ABNT NBR-5739:2007 [10] y ABNT NBR-7215:1996 [11].

\subsection{Etapa I: Rumbo al UHPFRC}

Para el diseño de los tratamientos se establecen cuatro factores controlables (i) A: edad de la muestra, con los niveles de control A1 de 7 días, A2 de 14 días y A3 de 28 días; (ii) B: tamaño máximo de la escoria, con los niveles de control B1 de $870 \mu \mathrm{m}$, B2 de $1700 \mu \mathrm{m}$ y B3 de $1180 \mu \mathrm{m}$; (iii) C: tipo de mezcla, con los niveles de control C1 para UHPRC-1, C2 para UHPRC-2 y C3 para UHPRC-3; y (iv) D: tipo de curado, con los niveles de control D1 o CTT (con tratamiento térmico de curado) y D2 o STT (sin tratamiento térmico de curado).

Todas las posibles combinaciones conducen a 54 experimentos que deben ser introducidos en el diseño, para tres repeticiones en cada caso tenemos un total de 162 cuerpos de prueba. La relación a/a se mantuvo fija en 0,15 y la relación a/c fue de 0,31 para UHPRC-1, de 0,28 para UHPRC-2 y de 0,24 para UHPRC3. La cantidad de arena de sílice, superplastificante y agua se mantuvieron constantes. Los tres tipos de mezclas son diferenciadas por los porcentajes de aglomerantes en cada caso, (Tabla 1).

\subsection{Etapa II: UHPFRC con fibras largas producidas en Brasil}

En el diseño de los tratamientos se establecen cuatro factores controlables (i) A: edad de la muestra, con los niveles de control A1 de 7 días, A2 de 28 días y A3 de 90 días; (ii) B: tipo de mezcla, con los niveles de control B1 para UHPRC-1, B2 para UHPRC-2, B3 para UHPRC-3 y B4 para UHPFRC-4; (iii) C: tipo de fibras, con los niveles de control $\mathrm{C} 1$ sin fibras, $\mathrm{C} 2$ con $1 \%$ de fibras de polipropileno y $\mathrm{C} 3$ con $1 \%$ de fibras largas de acero; y (iv) D: tipo de curado, con los niveles de control D1 o CTT y D2 o STT.

Todas las posibles combinaciones conducen a 72 experimentos que deben ser introducidas en el diseño, si se consideran tres repeticiones tenemos un total de 216 cuerpos de prueba. La relación a/a se mantuvo fija en 0,15 y la relación a/c fue de 0,24 para UHPRC-1, de 0,22 para UHPRC-2 y de 0,21 para UHPRC-3 y UHPFRC-4. Los tres tipos de mezclas son diferenciadas por los porcentajes de aglomerantes en cada caso, (Tabla 1), la diferencia entre las mezclas 3 y 4 es el tipo de granulometría de la escoria.

\subsection{Etapa III: UHPFRC con fibras cortas producidas en China}

En el diseño de los tratamientos se establecen tres factores controlables (i) A: edad de la muestra, con los niveles de control A1 de 7 días, A2 de 28 días y A3 de 90 días; (ii) B: tamaño del cilindro, con los niveles de control B1 o CP-5 que corresponde a cilindros con $5 \mathrm{~cm}$ de diámetro y $10 \mathrm{~cm}$ de altura, B2 o CP-7,5 que corresponde a cilindros con 7,5 cm de diámetro y $15 \mathrm{~cm}$ de altura; y (iii) $\mathrm{C}$ : porcentaje de fibras, con los niveles de control $\mathrm{C} 1$ con $0 \%, \mathrm{C} 2$ con $1 \%$ y C3 con $1,5 \%$ de fibras cortas de acero de $13 \mathrm{~mm}$ de longitud y $0,2 \mathrm{~mm}$ de diámetro.

Todas las posibles combinaciones conducen a 24 experimentos que deben ser introducidas en el diseño, si se consideran tres repeticiones tenemos un total de 72 cuerpos de prueba. La relación a/a se mantuvo fija en 0,13 y la relación a/c se mantuvo fija en 0,19. Se incluyó polvo de cuarzo en la mezcla, (Tabla 1).

\section{RESULTADOS}

\subsection{Etapa I: Rumbo al UHPFRC}

La Tabla 2 muestra que el mayor valor de resistencia a la compresión promedio fue de 138,12 MPa obtenido para la mezcla A3B1C3D1, resaltada en la Tabla 2, la cual tiene un desvío padrón $(\sigma)$ de $\pm 3,23$. Fue observado que a los 28 días de curado la mezcla A3B2C1D1, resaltada en la Tabla 2, disminuyó en $27 \%$ la resistencia alcanzada a los 7 días de curado, lo cual puede ser atribuido a errores involuntarios en la elaboración de los cuerpos de prueba.

En promedio a los 7 días de curado se alcanzó un $78 \%$ de la resistencia de los 28 días. En la Tabla 3 es posible observar que el desvío relativo máximo es menor al 6\% en todas las mezclas de concreto, cumpliendo con las especificaciones de la norma ABNT NBR-7215:1996 [11].

En la Tabla 4 se observan los resultados del análisis de varianza usando ANOVA, para todos los factores controlables y las combinaciones entre ellos reflejados en la columna llamada Fuente, notamos que la probabilidad fue igual a cero, esto quiere decir que la influencia de cada factor controlable es significativo 
sobre la variable de respuesta, que en nuestro caso es la resistencia a la compresión.

Tabla 2: Resistencia a la compresión promedio a los 7 y 28 días, Etapa I (MPa) Fuente propia.

\begin{tabular}{|c|c|c|c|c|c|c|c|c|c|}
\hline & \multicolumn{9}{|c|}{7 Dias } \\
\hline & \multicolumn{3}{|c|}{ B1 (GGBS-1) } & \multicolumn{3}{|c|}{ B2 (GGBS-2) } & \multicolumn{3}{|c|}{ B3 (GGBS-3) } \\
\hline & C1 & $\mathrm{C} 2$ & C3 & C1 & $\mathrm{C} 2$ & C3 & C1 & $\mathrm{C} 2$ & C3 \\
\hline D1 & 51,37 & 83,61 & 105,64 & 62,88 & 80,67 & 89,22 & 55,57 & 73,90 & 118,90 \\
\hline $\mathrm{D} 2$ & 60,00 & 94,69 & 92,80 & 70,12 & 68,39 & 86,37 & 78,01 & 77,08 & 95,33 \\
\hline \multirow{5}{*}{$\sigma$} & 1,60 & 0,53 & 3,03 & 0,97 & 1,68 & 0,76 & 1,39 & 2,31 & 0,76 \\
\hline & 0,67 & 0,77 & 4,72 & 1,74 & 0,88 & 1,24 & 2,68 & 1,60 & 2,03 \\
\hline & \multicolumn{9}{|c|}{28 Dias } \\
\hline & \multicolumn{3}{|c|}{ B1 (GGBS-1) } & \multicolumn{3}{|c|}{ B2 (GGBS-2) } & \multicolumn{3}{|c|}{ B3 (GGBS-3) } \\
\hline & C1 & $\mathrm{C} 2$ & C3 & C1 & $\mathrm{C} 2$ & C3 & C1 & $\mathrm{C} 2$ & $\mathrm{C} 3$ \\
\hline D1 & 71,76 & 112,82 & 138,12 & 46,05 & 98,48 & 121,74 & 89,98 & 101,55 & 134,98 \\
\hline $\mathrm{D} 2$ & 80,63 & 116,37 & 121,51 & 94,07 & 105,91 & 114,90 & 110,13 & 99,97 & 117,37 \\
\hline \multirow{2}{*}{$\sigma$} & 1,18 & 0,97 & 3,23 & 1,85 & 4,07 & 1,10 & 3,76 & 3,79 & 0,66 \\
\hline & 1,43 & 0,58 & 2,12 & 0,25 & 3,64 & 1,58 & 1,87 & 2,50 & 4,64 \\
\hline
\end{tabular}

Tabla 3: Planilla de residuos, Etapa I. Fuente propia.

\begin{tabular}{|c|c|c|c|c|c|c|c|c|c|c|c|c|c|c|c|c|c|c|}
\hline \multirow{2}{*}{\multicolumn{4}{|c|}{$\begin{array}{l}\text { A1 } 7 \text { Dias } \\
\text { B1 (GGBS-1) }\end{array}$}} & & & & & & & \multicolumn{9}{|c|}{ A3 28 Dias } \\
\hline & & & & \multicolumn{3}{|c|}{ B2 (GGBS-2) } & \multicolumn{3}{|c|}{ B3 (GGBS-3) } & \multicolumn{3}{|c|}{ B1 (GGBS-1) } & \multicolumn{3}{|c|}{ B2 (GGBS-2) } & \multicolumn{3}{|c|}{ B3 (GGBS-3) } \\
\hline & $\overline{\mathrm{C} 1}$ & $\mathrm{C} 2$ & $\mathrm{C3}$ & C1 & C2 & $\mathrm{C3}$ & C1 & $\mathrm{C} 2$ & $\mathrm{C3}$ & C1 & C2 & $\mathrm{C3}$ & C1 & $\mathrm{C} 2$ & $\mathrm{C3}$ & $\mathrm{C} 1$ & C2 & $\mathrm{C3}$ \\
\hline \multirow[t]{3}{*}{ D1 } & $-1,09$ & $-0,61$ & 3,43 & $-0,93$ & $-1,84$ & 0,50 & $-1,11$ & 2,62 & 0,76 & 1,08 & $-1,01$ & $-2,24$ & $-2,01$ & $-4,14$ & 0,61 & $-4,34$ & 0,68 & 0,76 \\
\hline & $-0,76$ & 0,30 & $-2,30$ & 1,01 & 0,41 & 0,37 & $-0,44$ & $-0,86$ & 0,01 & 0,18 & 0,08 & $-1,46$ & 0,37 & 0,15 & $-1,27$ & 2,05 & $-4,08$ & $-0,36$ \\
\hline & 1,84 & 0,32 & $-1,14$ & $-0,08$ & 1,43 & $-0,87$ & 1,56 & $-1,76$ & $-0,76$ & $-1,25$ & 0,93 & 3,70 & 1,64 & 3,99 & 0,66 & 2,29 & 3,41 & $-0,40$ \\
\hline \multirow[t]{3}{*}{ D2 } & $-0,58$ & 0,46 & $-5,25$ & $-1,57$ & $-0,07$ & $-1,42$ & 1,24 & 1,85 & 1,47 & 0,24 & 0,60 & $-1,18$ & $-0,22$ & 2,60 & $-1,80$ & 1,96 & 1,31 & $-4,74$ \\
\hline & 0,73 & $-0,89$ & 3,88 & $-0,31$ & 0,91 & 0,86 & 1,84 & $-0,87$ & 0,85 & $-1,54$ & $-0,05$ & 2,44 & $-0,05$ & 1,55 & 0,66 & $-0,21$ & $-2,88$ & 0,20 \\
\hline & $-0,15$ & 0,43 & 1,37 & 1,87 & $-0,84$ & 0,56 & $-3,07$ & $-0,98$ & $-2,32$ & 1,30 & $-0,55$ & $-1,27$ & 0,27 & $-4,16$ & 1,15 & $-1,76$ & 1,57 & 4,53 \\
\hline
\end{tabular}

Tabla 4: Planilla ANOVA, Etapa I. Fuente propia.

\begin{tabular}{llllll}
\hline FUENTE & SQ & \multicolumn{2}{l}{ GDL MQ } & TESTE F & PROBABILIDAD \\
\hline $\mathrm{A}$ & $16.520,83$ & 2 & $8.260,41$ & $1.481,14$ & 0,00 \\
\hline $\mathrm{B}$ & $2.964,79$ & 2 & $1.482,40$ & 265,80 & 0,00 \\
\hline $\mathrm{C}$ & $39.614,43$ & 2 & $19.807,22$ & $3.551,56$ & 0,00 \\
\hline $\mathrm{D}$ & 639,37 & 1 & 639,37 & 114,64 & 0,00 \\
\hline $\mathrm{AB}$ & 185,37 & 4 & 46,34 & 8,31 & 0,00 \\
$\mathrm{AC}$ & 444,46 & 4 & 111,12 & 19,92 & 0,00 \\
$\mathrm{AD}$ & 321,77 & 2 & 160,89 & 28,85 & 0,00 \\
$\mathrm{BC}$ & $5.414,05$ & 4 & $1.353,51$ & 242,69 & 0,00 \\
$\mathrm{BD}$ & 792,85 & 2 & 396,43 & 71,08 & 0,00 \\
$\mathrm{CD}$ & $6.960,87$ & 2 & $3.480,43$ & 624,06 & 0,00 \\
$\mathrm{ABC}$ & $2.502,00$ & 8 & 312,75 & 56,08 & 0,00 \\
$\mathrm{BCD}$ & 657,59 & 4 & 164,40 & 29,48 & 0,00 \\
$\mathrm{CDA}$ & 720,56 & 4 & 180,14 & 32,30 & 0,00 \\
$\mathrm{DAB}$ & $1.425,63$ & 4 & 356,41 & 63,91 & 0,00 \\
$\mathrm{ABCD}$ & $1.368,45$ & 8 & 171,06 & 30,67 & 0,00 \\
\cline { 1 - 3 } Erro & 602,32 & 108 & 5,58 & & \\
\cline { 1 - 2 } TOTAL= & $81.135,36$ & 161 & &
\end{tabular}

En la Figura 3 notamos que el factor controlable $C$ referente al diseño de mezcla tiene un $49 \%$ de importancia en la resistencia a la compresión, el factor controlable A referente a la edad de la muestra tiene un $20 \%$ de importancia, las combinaciones CD referentes al diseño de mezcla junto al tratamiento térmico en el curado y BC referentes a la granulometría de la escoria junto con el diseño de mezcla poseen una importancia de 9 y $7 \%$ respectivamente. 


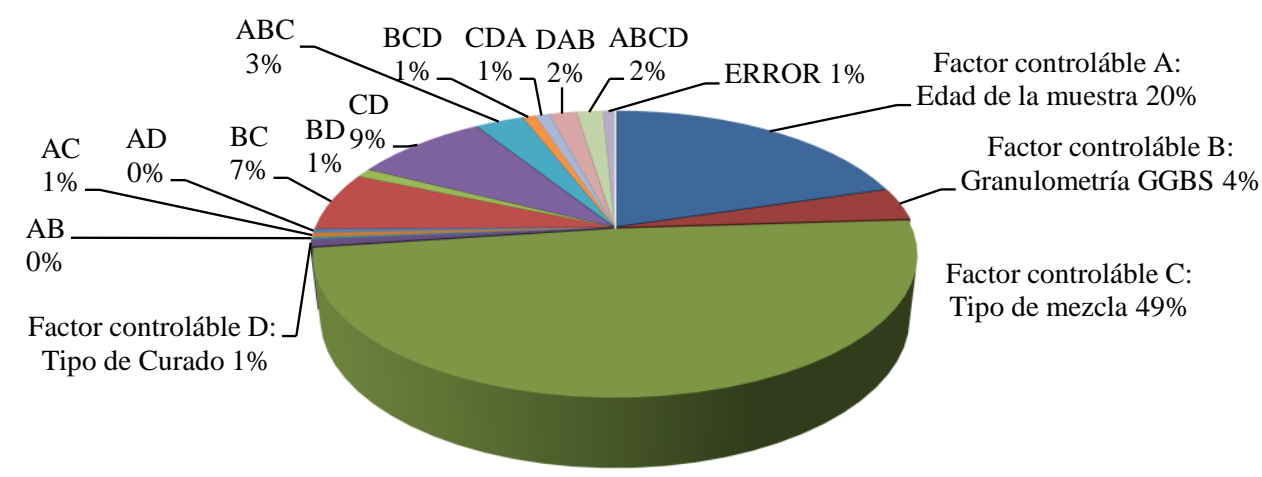

Figura 3: Resultados ANOVA - Etapa I. Fuente propia.

\subsection{Etapa II: UHPFRC con fibras largas producidas en Brasil}

En la Tabla 5 se observa que el mayor valor de resistencia a la compresión promedio fue de 132,17 MPa obtenido para la mezcla A2B3C3D1, con un desvío padrón $(\sigma)$ de $\pm 2,96$. La mayor resistencia obtenida en esta etapa fue menor que la resistencia obtenida en la etapa anterior. En esta etapa se usaron probetas cilíndricas de mayor tamaño a causa de la longitud de las fibras nacionales disponibles.

Tabla 5: Resistencia a la compresión promedio a los 7 y 28 días, Etapa II (MPa). Fuente propia.

\begin{tabular}{|c|c|c|c|c|c|c|c|c|c|c|c|c|}
\hline & \multicolumn{12}{|c|}{ A1 7 Dias } \\
\hline & \multicolumn{3}{|c|}{ B1: UHPFRC-1 } & \multicolumn{3}{|c|}{ B2: UHPFRC-2 } & \multicolumn{3}{|c|}{ B3: UHPFRC-3 } & \multicolumn{3}{|c|}{ B4: UHPFRC-4 } \\
\hline & C1-S & C2-P & C3-A & C1-S & C2-P & C3-A & C1-S & C2-P & C3-A & C1-S & C2-P & C3-A \\
\hline D1 & 104,88 & 89,48 & 121,83 & 96,97 & 93,08 & 102,80 & 120,44 & 99,17 & 130,77 & 117,11 & 103,51 & 121,94 \\
\hline D2 & 85,94 & 65,26 & 88,07 & 70,91 & 81,00 & 77,70 & 91,98 & 71,45 & 98,85 & 84,54 & 76,67 & 81,53 \\
\hline \multirow{5}{*}{$\sigma$} & 0,80 & 0,91 & 2,37 & 1,78 & 2,74 & 2,04 & 0,89 & 4,10 & 0,62 & 3,96 & 2,27 & 3,53 \\
\hline & 1.50 & 2.27 & 1.17 & 0.82 & 1.35 & 1.55 & 1.51 & 1.10 & 2.31 & 3.96 & 3.54 & 2.58 \\
\hline & $\mathrm{A} 2$ & 8 Dias & & & & & & & & & & \\
\hline & \multicolumn{3}{|c|}{ B1: UHPFRC-1 } & \multicolumn{3}{|c|}{ B2: UHPFRC-2 } & \multicolumn{3}{|c|}{ B3: UHPFRC-3 } & \multicolumn{3}{|c|}{ B4: UHPFRC-4 } \\
\hline & C1-S & C2-P & C3-A & C1-S & $\mathrm{C} 2-\mathrm{P}$ & C3-A & C1-S & C2-P & C3-A & C1-S & $\mathrm{C} 2-\mathrm{P}$ & C3-A \\
\hline D1 & 108,81 & 99,19 & 118,90 & 106,16 & 85,17 & 124,83 & 104,73 & 90,61 & 132,17 & 121,69 & 106,31 & 126,80 \\
\hline D2 & 92,46 & 88,02 & 107,46 & 91,29 & 58,08 & 104,83 & 74,27 & 70,67 & 113,29 & 84,54 & 83,95 & 92,52 \\
\hline \multirow[t]{2}{*}{$\sigma$} & 3,37 & 2,01 & 1,16 & 4,97 & 3,73 & 2,77 & 3,40 & 2,33 & 2,96 & 0,67 & 1,63 & 1,29 \\
\hline & 2,74 & 2,99 & 2,48 & 4,85 & 2,08 & 4,40 & 3,68 & 2,05 & 0,62 & 1,20 & 0,84 & 2,76 \\
\hline
\end{tabular}

Tabla 6: Planilla de residuos, Etapa II (MPa). Fuente propia.

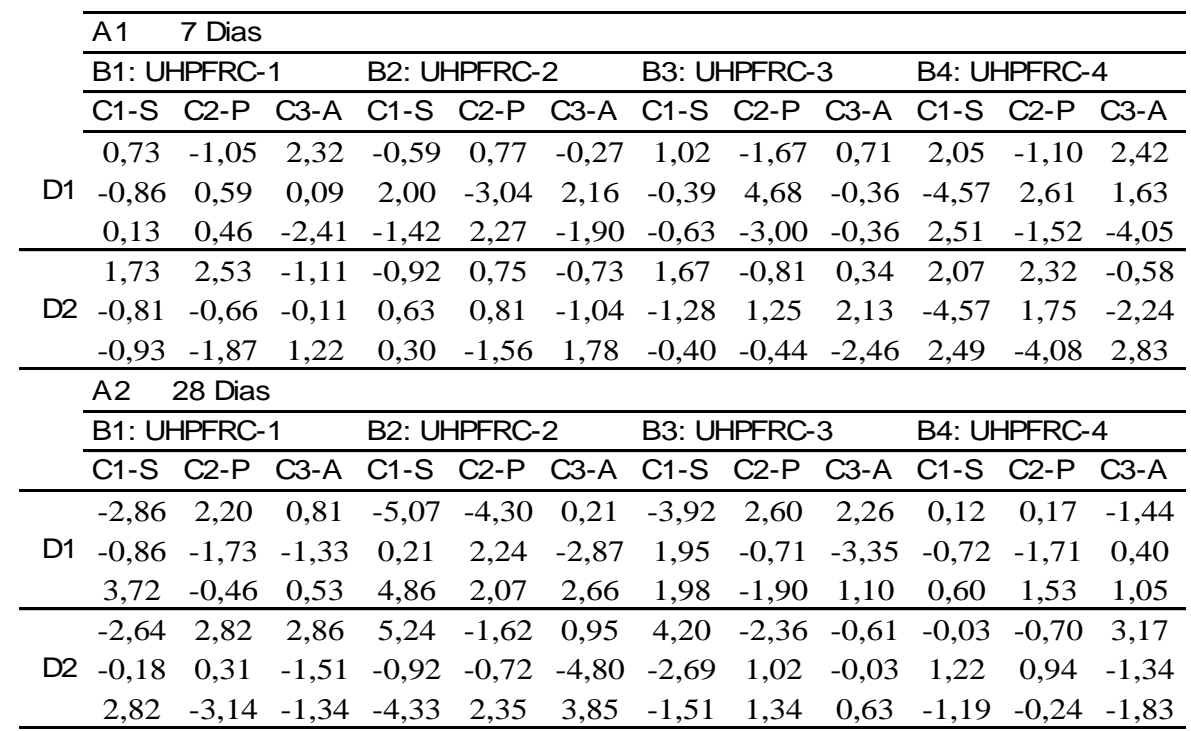

Comparando los resultados de resistencia a la compresión entre los 7 y 28 días notamos que algunas 
mezclas en lugar de aumentar su resistencia la disminuyeron, es el caso de las mezclas A2B1C3D1 que disminuyó en un 2,4\%; $\mathrm{A} 2 \mathrm{~B} 2 \mathrm{C} 2 \mathrm{D} 1$ y $\mathrm{A} 2 \mathrm{~B} 2 \mathrm{C} 2 \mathrm{D} 2$ en un 8,5 y $28,3 \%$ respectivamente; A2B3C1D1 y $\mathrm{A} 2 \mathrm{~B} 3 \mathrm{C} 1 \mathrm{D} 2$ en un 13,04 y $19,25 \%$ respectivamente; y para A2B3C2D1 y A2B3C2D2 en un 8,63 y 1,1\% respectivamente, lo cual puede ser atribuido a errores involuntarios en la elaboración de los cuerpos de prueba $o$ a problemas acontecidos en el laboratorio para mantener fija la temperatura del baño térmico en el curado. En promedio a los 7 días de curado se alcanzó un $92 \%$ de la resistencia de los 28 días.

La Tabla 6 muestra que el desvío relativo máximo es menor al $6 \%$ en todas las mezclas de concreto, cumpliendo con las especificaciones de la norma ABNT NBR-7215:1996 [11].

El análisis de varianza mostrado en la Tabla 7 indicó que todos los factores controlables tienen influencia significativa en la variable de respuesta, en nuestro caso en la resistencia a la compresión, siendo de mayor la importancia la actuación de los factores tipo de curado con un 29\%, tipo de fibra con un $23 \%$ y edad de la muestra con 19\% como puede ser observado en la Figura 4.

Aplicar tratamiento térmico en el curado acelera la resistencia en edades tempranas, por tanto de aquí en adelante ese factor pasa a ser constante. La Figura 4 también muestra que el factor controlable $\mathrm{C}$ referente al tipo de trazo solo tiene un $2 \%$ de importancia sobre la variable de respuesta, por tanto de aquí en adelante este factor también pasa a ser constante.

Tabla 7: Planilla ANOVA, Etapa II. Fuente propia.

\begin{tabular}{llllll}
\hline FUENTE & SQ & GDL MQ & TESTE F & PROBABILIDAD \\
\hline A & $14.782,43$ & 2 & $7.391,21$ & 894,57 & 0,00 \\
\hline B & $1.356,42$ & 3 & 452,14 & 54,72 & 0,00 \\
\hline C & $17.578,10$ & 2 & $8.789,05$ & $1.063,75$ & 0,00 \\
\hline D & $22.490,70$ & 1 & $22.490,70$ & $2.722,08$ & 0,00 \\
\hline AB & $3.743,44$ & 6 & 623,91 & 75,51 & 0,00 \\
AC & $1.280,81$ & 4 & 320,20 & 38,75 & 0,00 \\
AD & $2.217,85$ & 2 & $1.108,93$ & 134,21 & 0,00 \\
BC & $1.827,77$ & 6 & 304,63 & 36,87 & 0,00 \\
BD & $1.538,03$ & 3 & 512,68 & 62,05 & 0,00 \\
CD & 539,03 & 2 & 269,52 & 32,62 & 0,00 \\
ABC & $3.647,54$ & 12 & 303,96 & 36,79 & 0,00 \\
BCD & $1.016,78$ & 6 & 169,46 & 20,51 & 0,00 \\
CDA & 251,69 & 4 & 62,92 & 7,62 & 0,00 \\
DAB & 937,28 & 6 & 156,21 & 18,91 & 0,00 \\
ABCD & $2.025,95$ & 12 & 168,83 & 20,43 & 0,00 \\
\hline Error & $1.189,77$ & 144 & 8,26 & & \\
$=$ & $76.423,59$ & 215 & &
\end{tabular}

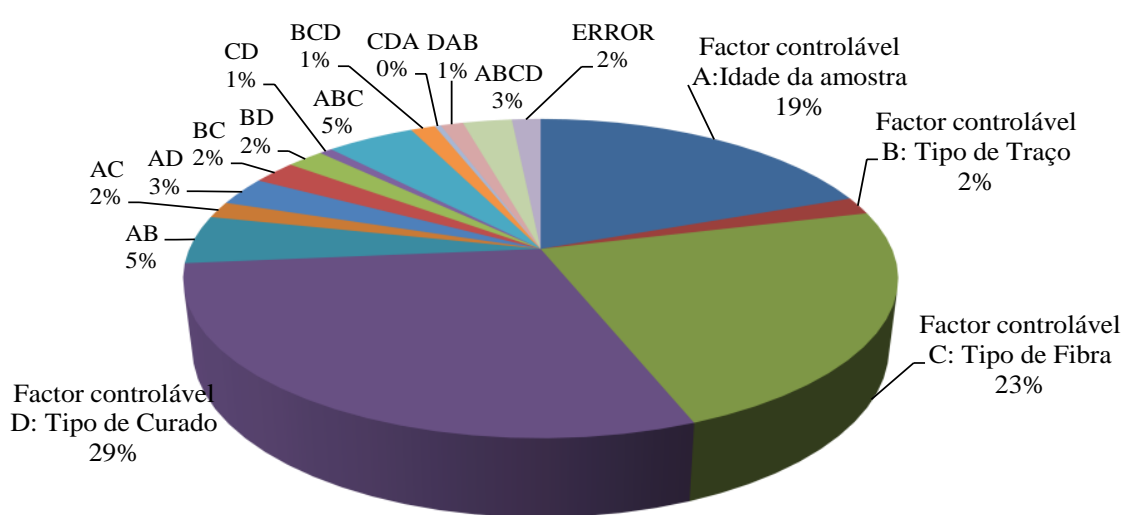

Figura 4: Resultados ANOVA - Etapa II. Fuente propia.

\subsection{Etapa III: UHPFRC con fibras cortas producidas en China}

La Tabla 8 muestra que el mayor valor de resistencia a la compresión promedio fue de 155,29 MPa obtenido para la mezcla A2B1C2 y de 153,44 MPa para la mezcla A2B2C3, con desvíos padrón $(\sigma)$ de $\pm 3,10$ y $\pm 5,07$ respectivamente. Esos valores son superiores a $150 \mathrm{MPa}$, que es el valor de resistencia a la compresión objetivo de este estudio. 
Por otro lado, observando los otros diseños de mezcla de la Tabla 8 y comparando los valores de resistencia a la compresión entre los 7 y 28 días de curado observamos que algunos casos presentan una disminución de la resistencia, específicamente las mezclas A2B1C3 y A2B2C1, cuyos valores de resistencia disminuyeron a los 28 días en un 6,5 y 7,7\% respectivamente. Esto puede ser atribuido a errores en la elaboración de los cuerpos de prueba o a problemas acontecidos en el laboratorio para mantener fija la temperatura del baño térmico en el curado.

Otro aspecto importante de señalar es que en promedio a los 7 días de curado se alcanzó un 94\% de la resistencia a la compresión de los 28 días de curado, es por ello que en la Figura 5 el factor controlable relativo a la edad de la muestra es del 4\%. En la Tabla 9 observamos que el desvío relativo máximo es menor al 6\% en todas las mezclas de concreto, cumpliendo con las especificaciones de la norma ABNT NBR7215:1996 [11]. El análisis de varianza mostrado en la Tabla 10 indica que todos los factores controlables tienen influencia significativa en la variable de respuesta, en nuestro caso en la resistencia a la compresión, siendo de mayor importancia la actuación del factor controlable $\mathrm{C}$ referente al porcentaje de fibras, con un valor de $40 \%$. El factor controlable combinado entre ABC, es decir, entre la edad de la muestra, tamaño del espécimen y porcentaje de fibra, alcanzó un $21 \%$ de importancia, como se refleja en la Figura 5.

Tabla 8: Resistencia a la compresión promedio a los 7 y 28 días, Etapa III (MPa). Fuente propia.

\begin{tabular}{|c|c|c|c|c|c|}
\hline \multicolumn{6}{|c|}{$\begin{array}{ll}\text { A1 } & 7 \text { Dias }\end{array}$} \\
\hline \multicolumn{3}{|c|}{$\mathrm{B} 1(\mathrm{CP}=5 \mathrm{~cm})$} & \multicolumn{3}{|c|}{$\mathrm{B} 2(\mathrm{CP}=7,5 \mathrm{~cm})$} \\
\hline C1 (0\%) & C2 (1\%) & C3 $(1,5 \%)$ & C1 (0\%) & C2 (1\%) & C3 $(1,5 \%)$ \\
\hline 130,36 & 148,56 & 145,47 & 128,70 & 122,87 & 132,44 \\
\hline$\sigma \overline{5,63}$ & 2,50 & 4,54 & 2,99 & 5,00 & 0,85 \\
\hline \multicolumn{6}{|c|}{ A2 28 Dias } \\
\hline \multicolumn{3}{|c|}{$\mathrm{B} 1(\mathrm{CP}=5 \mathrm{~cm})$} & \multicolumn{3}{|c|}{$\mathrm{B} 2(\mathrm{CP}=7,5 \mathrm{~cm})$} \\
\hline C1 (0\%) & C2 (1\%) & C3 $(1,5 \%)$ & C1 (0\%) & $\mathrm{C} 2(1 \%)$ & C3 $(1,5 \%)$ \\
\hline 130,42 & 155,29 & 135,99 & 118,76 & 148,47 & 153,44 \\
\hline$\sigma \overline{4,80}$ & 3,10 & 3,24 & 0,56 & 2,61 & 5,07 \\
\hline
\end{tabular}

Tabla 9: Planilla de residuos, Etapa III. Fuente propia.

\begin{tabular}{|c|c|c|c|c|c|}
\hline \multicolumn{6}{|c|}{$\begin{array}{ll}\text { A1 } & 7 \text { Dias }\end{array}$} \\
\hline \multicolumn{3}{|c|}{$\mathrm{B} 1(\mathrm{CP}=5 \mathrm{~cm})$} & \multicolumn{3}{|c|}{$\mathrm{B} 2(\mathrm{CP}=7,5 \mathrm{~cm})$} \\
\hline C1 (0\%) & $\mathrm{C} 2(1 \%)$ & C3 $(1,5 \%)$ & C1 $(0 \%)$ & $\mathrm{C} 2(1 \%)$ & C3 $(1,5 \%)$ \\
\hline$-5,44$ & $-2,11$ & 4,83 & 0,21 & $-4,49$ & 0,85 \\
\hline 5,80 & $-0,66$ & $-4,17$ & $-3,08$ & $-0,90$ & $-0,01$ \\
\hline$-0,36$ & 2,76 & $-0,66$ & 2,88 & 5,38 & $-0,85$ \\
\hline \multicolumn{6}{|c|}{ A2 28 Dias } \\
\hline \multicolumn{3}{|c|}{$\mathrm{B} 1(\mathrm{CP}=5 \mathrm{~cm})$} & \multicolumn{3}{|c|}{$\mathrm{B} 2(\mathrm{CP}=7,5 \mathrm{~cm})$} \\
\hline C1 (0\%) & $\mathrm{C} 2(1 \%)$ & C3 $(1,5 \%)$ & C1 $(0 \%)$ & C2 (1\%) & C3 $(1,5 \%)$ \\
\hline$-4,74$ & 0,64 & 0,43 & 0,51 & $-0,94$ & $-4,28$ \\
\hline$-0,13$ & 2,72 & 3,01 & 0,21 & $-2,01$ & $-1,33$ \\
\hline 4,87 & $-3,37$ & $-3,43$ & $-0,58$ & 2,95 & 5,60 \\
\hline
\end{tabular}

Tabla 10: Planilla ANOVA, Etapa III. Fuente propia.

\begin{tabular}{llllll}
\hline \multicolumn{2}{l}{ FUENTE SQ } & \multicolumn{2}{l}{ GDIMQ } & \multicolumn{2}{l}{ TESTE F PROBABILIDAD } \\
\hline A & 309,86 & 2 & 154,93 & 4,30 & 0,02 \\
$\mathrm{~B}$ & 210,13 & 1 & 210,13 & 5,84 & 0,02 \\
$\mathrm{C}$ & $2.642,23$ & 2 & $1.321,11$ & 36,70 & 0,00 \\
$\mathrm{AB}$ & 623,18 & 2 & 311,59 & 8,66 & 0,00 \\
$\mathrm{AC}$ & 727,28 & 4 & 181,82 & 5,05 & 0,00 \\
$\mathrm{BC}$ & 131,74 & 2 & 65,87 & 1,83 & 0,18 \\
$\mathrm{ABC}$ & $1.397,08$ & 4 & 349,27 & 9,70 & 0,00 \\
\hline Erro & 501,47 & 36 & 13,93 & \\
\cline { 1 - 1 } \\
TOTAL $=$ & $6.542,98$ & 53 &
\end{tabular}




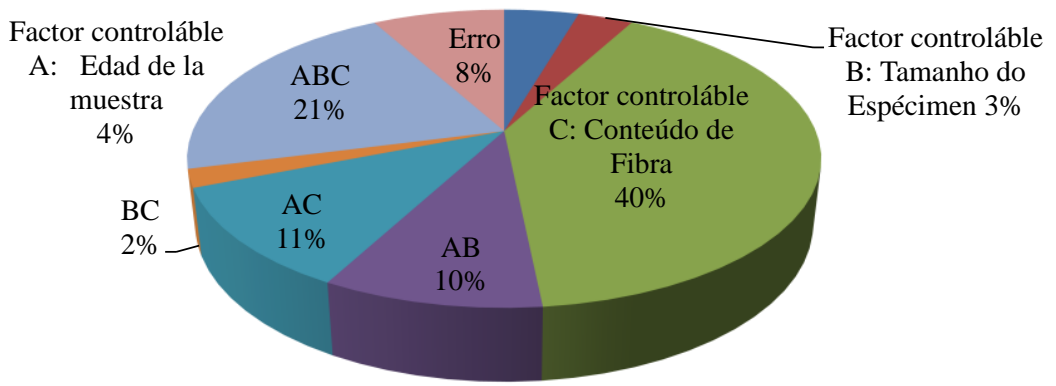

Figura 5: Resultados ANOVA - Etapa III. Fuente propia.

\section{DISCUSIÓN}

El análisis de varianza realizado en la primera etapa del trabajo indicó que todos los factores controlables tienen influencia significativa en la variable de respuesta que en nuestro caso es la resistencia a la compresión, siendo de mayor importancia la actuación del factor C (tipo de mezcla) con un 49\%, el factor A (edad de la muestra) con un $20 \%$ y la combinación CD (tipo de mezcla con tipo de curado) con $9 \%$.

Analizando la influencia del factor controlable B referente a la granulometría de la escoria, se observa que esa variable alcanzó un $4 \%$ de importancia, siendo las granulometrías con tamaños máximos menores (B1 y B3) las que arrojaron los mejores resultados de resistencia a los 28 días, por ese motivo en la próxima etapa la granulometría de la escoria pasó a ser un factor constante. El mayor valor de la resistencia promedio obtenido fue de 138,12 MPa con el diseño de mezcla C3, por tal motivo es necesario incrementar el valor de la resistencia en al menos un $9 \%$ para alcanzar la meta de los $150 \mathrm{MPa}$.

Larrard-Sedran [12] y también Richard-Cheyrezy [13] utilizaron el empaquetamiento de partículas para densificar la matriz e incrementar la resistencia, basado en eso se decide evaluar la distribución de las partículas en el diseño de mezcla C3 usando el programa EMMA [34]. Al comparar la curva experimental con la teórica que tiene EMMA, es posible visualizar una deficiencia de partículas con tamaños menores a $10 \mu \mathrm{m}$, es decir, se hizo evidente que la compensación de esas partículas densificaría la matriz y por tanto incrementaría la resistencia. Sin embargo obtener esa granulometría implica un minucioso trabajo de molienda y distribución del material, lo cual se aleja del tan deseado procedimiento práctico. Por tanto se decide establecer 0,6 mm como tamaño máximo del material, observando una mejoría en el acoplamiento de las curvas teórica y experimental con un simple tamizado del material. También se decide incluir las fibras como un factor controlable en la siguiente etapa de la pesquisa, siguiendo lo planteado por algunos autores como Rossi et al. [14], Lim-Nawy [15], Graybeal [17], Thanh [18] y Kang et al. [26].

Los resultados obtenidos en la segunda etapa no superaron a los obtenidos en la etapa anterior a los 28 días de curado. Las fibras no contribuyeron con el incremento de la resistencia, posiblemente por no presentar un tamaño adecuado en relación al diámetro de los cilindros usados. Los valores menores y mayores de la resistencia en esta etapa fueron obtenidos usando fibras de polipropileno y de acero, respectivamente. Por ello se decide para la tercera etapa incluir como factor controlable al tamaño de los cuerpos de prueba y un tipo de fibra adecuada a la escala de los especímenes y a las indicaciones de la bibliografía consultada [12, 13, 16, 26]. El análisis de varianza usando ANOVA en la etapa II demostró que el tipo de curado es de gran importancia para acelerar la resistencia en los cuerpos de prueba, por lo tanto se decide aplicar tratamiento térmico en todos los especímenes de la etapa siguiente, también ANOVA en esta etapa demostró que el tipo de diseño de mezcla no fue significativo sobre la resistencia a la compresión, por lo tanto este factor se considerará constante.

En la tercera y última etapa la resistencia a la compresión se incrementó significativamente, superando los $150 \mathrm{MPa}$ que definen al concreto como UHPFRC. El porcentaje de fibras demostró tener gran importancia en los resultados, lo cual contradice los resultados mostrados por Hoang y Fehling [28]. Por otro lado, los resultados obtenidos en este estudio se asemejan a los alcanzados por la mayoría de los pesquisadores consultados, como por ejemplo Kahanji et al. [29], Wu et al. [30] y Alsalman et al. [31].

\section{CONCLUSIONES}

Resistencia a la compresión promedio de 155,29 MPa fue obtenida con una mezcla de concreto que atiende las siguientes características: (i) relación agua/cemento de 0,19; (ii) relación agua/aglomerantes de 0,13; (iii) inclusión del 1,5\% de fibras cortas de acero; (iv) uso de 26\% de residuos industriales (GGBS y humo de sílice) en sustitución parcial del cemento. 
Resistencia a la compresión promedio de 138,12 MPa fue obtenida con una mezcla de concreto que atiende las siguientes características: (i) relación agua/cemento de 0,24; (ii) relación agua/aglomerantes de 0,15; (iii) sin la inclusión de fibras; (iv) uso de 35\% de residuos industriales (GGBS y humo de sílice) en sustitución parcial del cemento.

El tratamiento térmico en el proceso de curado de los cuerpos de prueba aceleró la ganancia de resistencia a la compresión en edades tempranas.

El análisis de varianza usando ANOVA demostró ser un método eficiente para determinar la importancia de cada factor controlable sobra la variable de respuesta, que en nuestro caso fue la resistencia a la compresión, lo cual contribuyó en la toma de decisiones en cada etapa experimental del estudio.

\section{AGRADECIMENTOS}

Los autores agradecen al laboratorio de Ensayos y Modelos Estructurales (LEME) por el apoyo brindado en la realización de este trabajo, muy especialmente a los técnicos de laboratorio Eurípedes Fontes y Edgar Wallace.

\section{BIBLIOGRAFIA}

[1] LOPEZ VILLAMARÍN, M. A., OSORIO GAMBOA, D., Influencia del proceso de fabricación sobre las propiedades mecánicas de los concretos de ultra alto desempeño (UHPC), Trabajo de grado, Maestría en Ingeniería Civil, Facultad de Ingeniería Pontificia Universidad Javeriana, Bogotá D.C., Colombia, 2015.

[2] NIETO OSORIO, R. C., Análisis comparativo de concretos con aditivos reductores de agua: complementación utilizando el aditivo Glenium 3030 NS en concretos autonivelantes, Tesis en Ingeniería Civil, Facultad de Ingeniería Civil Universidad Nacional de Ingeniería, Lima, Perú, 2005.

[3] FERNANDEZ, C., Concretos de ultra alto desempeño tecnología y aplicaciones. Escuela de Ingeniería Civil, Universidad de Costa Rica, San José, Costa Rica, 2005.

[4] RODRIGO MAZIA, E. Reforço de pilares curtos de concreto armado por encamisamento com concreto de ultra-alto desempenho, Tese de Doutorado, Escola de Engenharia de São Carlos, Universidade de São Paulo, São Carlos, Brasil, 2017.

[5] TOLEDO, R., KOENDERS, E., FORMAGINI, S., et al., "Performance assessment of Ultra High Performance Fiber Reinforced Cementitious Composites in view of sustainability", Journal Materials and Design, v.36, pp.880-888, 2012

[6] MENDES, T., Efeito da nanossílica em matrizes cimentícias de ultra alta resistência, Tese de Doutorado, Centro Tecnológico, Universidade Federal de Santa Catarina, Florianópolis, Brasil, 2016.

[7] RESPLENDINO, J., TOULEMONDE, F., Designing and Building with UHPFRC, 1 ed., Wiley-ISTE, 2011.

[8] LÓPEZ, J., SERNA, P., CAMACHO, E., et al., "First Ultra-High-Performance Fibre-Reinforced Concrete Footbridge in Spain: Design and Construction", Journal Structural Engineering International, v.24, n.1, pp101-104.

[9] FEHLING, E., SCHMIDT, M., WALRAVEN, J., Ultra-High Performance Concrete Uhpc:Fundamentals, Design, Examples (Beton-Kalender Series), 5 ed., John Wiley \& Sons, 2015.

[10] ASSOCIAÇÃO BRASILEIRA DE NORMAS TÉCNICAS (2007), NBR 5739: Ensaio de compressão de corpos de prova cilíndricos. Rio de Janeiro, ABNT - Associação Brasileira de Normas Técnicas.

[11] ASSOCIAÇÃO BRASILEIRA DE NORMAS TÉCNICAS (1996), NBR 7215: Cimento Portland Determinação da Resistência à Compressão. Rio de Janeiro, ABNT - Associação Brasileira de Normas Técnicas.

[12] LARRARD, F., SEDRAN, T. "Optimization of Ultra-High-Performance Concrete by the use of a packing model”, Journal Cement and Concrete Research, v. 24, n. 1, pp. 997-1009, 1994.

[13] RICHARD, P., CHEYREZY, M. "Composition of reactive powder concretes", Journal Cement and Concrete Research, v. 25, pp. 1501-1511, 1995.

[14] ROSSI, P., ARCA, A., PARANT, E., et al., "Bending and compressive behaviors of a new cement composite”, Journal Cement and Concrete Research, v. 35, n. 1, pp. 27-33, 2005.

[15] LIM, D.H., NAWY, E. G. "Behavior of plain and steel-fiber-reinforced high-strength concrete under uniaxial and biaxial compression”, Journal Magazine of Concrete Research, v. 57, n. 10, pp. 603-610, 2005. 
[16] HABEL, K.; VIVIANI, M.; DENARIÉ, E.; BRÜHWILER, E. “Development of the mechanical properties of an Ultra-High Performance Fiber Reinforced Concrete (UHPFRC)", Journal Cement and Concrete Research, v.36, pp. 1362-1370, 2006.

[17] GRAYBEAL, B. "Compressive behavior of Ultra-High Performance Fiber Reinforced Concrete", Journal ACI Materials, v. 104, n. 2, pp. 146-152, 2007.

[18] THANH, LE., Ultra high performance fiber reinforced concrete paving flags, Ph.D Thesis, University of Liverpool, Liverpool, U.K., 2008.

[19] YANG, S.L., MILLARD, S.G., SOUTSOS, M.N., et al., "Influence of aggregate and curing regime on the mechanical properties of ultra-high performance fiber reinforced concrete (UHPFRC)", Journal Construction and Building Materials, v. 26, n. 6, pp. 2291-2298, 2009.

[20] TUAN, N., YE, G., BREUGEl, K., et al., "The study of using rice husk ash to produce ultra high performance concrete", Journal Construction and Building Materials, v. 25, pp. 2030-2035, 2011.

[21] HASSAN, A., JONES, S., MAHMUD, G. "Experimental test methods to determine the uniaxial tensile and compressive behavior of ultra-high performance fiber reinforced concrete (UHPFRC)", Journal Construction and Building Materials, v. 37, pp. 874-882, 2012.

[22] YU, R., SPIESZ, P., BROUWERS, H. "Mix design and properties assessment of Ultra High Performance Fiber Reinforced Concrete (UHPFRC)", Journal Cement and Concrete Research, v. 56, Pp. 29-39, 2014.

[23] WU, Z., SHI, C., KHAYAT, K. "Influence of silica fume content on microstructure development and bond to steel fiber in ultra-high strength cement based materials (UHSC)", Journal Cement and Concrete Composites, v. 71, Pp. 97-109, 2016.

[24] LAMPROPOULOS, A, PASCHALIS, S., TSIOULOU, O., et al., "Strengthening of reinforced concrete beams using ultra high performance fiber reinforced concrete (UHPFRC)", Journal Engineering Structures, v. 16, pp. 370-384, 2016.

[25] LIU, J., HAN, F., CUI, G., et al., "Combined effect of coarse aggregate and fiber on tensile behavior of ultra-high performance concrete", Journal Constructions and Building Materials, v. 121, pp. 310-318, 2016.

[26] KANG, S., CHOI, J., KOH, K., et al., "Hybrid effects of steel fiber and microfiber on the tensile behavior of ultra-high performance concrete", Journal Composites Structures, v. 145, pp. 37-42, 2016.

[27] LEE, J., HONG, S., JOH, CH., et al., "Biaxial tension-compression strength behaviour of UHPRC in plane elements", Journal Materials and Structures, v. 50, pp. 1-17, 2017.

[28] HOANG, A., FEHLING, E. "Influence of steel fiber content and aspect ratio on the uniaxial tensile and compressive behavior of ultra-high performance concrete", Journal Construction and Building Materials, v. 153, pp. 790-806, 2017.

[29] KAHANJI, Ch., ALI, F., NADJAI, A. "Structural performance of ultra-high performance fiberreinforced concrete beams", Journal Structural Concrete, v. 18, pp. 249-258, 2017.

[30] WU, Z., SHI, C., HE, W., et al., "Static and dynamic compressive properties of ultra-high performance concrete (UHPC) with hybrid steel fiber reinforcements", Journal Cement and Concrete Composites, n. 79, pp. +148-157, 2017.

[31] ALSALMAN, A., DANG, C., MICAH, W. "Development of ultra-high performance concrete with locally available materials", Journal Construction and Building Materials, v. 133, pp. 135-145, 2017.

[32] ASSOCIAÇÃO BRASILEIRA DE NORMAS TÉCNICAS (2003) NBR 5738: Concreto - Procedimento para moldagem e cura de corpos de prova. Rio de Janeiro, ABNT - Associação Brasileira de Normas Técnicas.

[33] ROJAS, R. Video “Produciendo UHPFRC”. Link: https://www.youtube.com/watch?v=MMhqZq2brdY

[34] EMMA. http://www.clubedoconcreto.com.br/2015/07/empacotamento-com-software-da-elkem-emma. html

\section{ORCID}

Rosangel Rojas

Christa Korzenowsky

Jose Yepez

Américo Campos Filho

Luiz Carlos Pinto da Silva https://orcid.org/0000-0003-3703-7328
https://orcid.org/0000-0003-0776-599X

https://orcid.org/0000-0001-6500-2910

https://orcid.org/0000-0002-2557-8779

https://orcid.org/0000-0001-7764-3010 
